\title{
Comparing students' understanding of Gauss's and Ampere's laws with field sources in square-like symmetries
}

\author{
Eder Hernandez, Esmeralda Campos, Pablo Barniol \\ Tecnologico de Monterrey, E. Garza Sada 2501, Monterrey, Mexico, 64849 \\ Genaro Zavala \\ Tecnologico de Monterrey, E. Garza Sada 2501, Monterrey, Mexico, 64849 \\ Universidad Andres Bello, Antonio Varas 880, Santiago, Chile
}

The research in electricity and magnetism has commonly focused on problem-solving abilities and the application of physical principles. Several studies have found that some concepts represent a challenge for undergraduate students, such as Gauss's and Ampere's laws. The inability to identify and analyze the symmetry of field and field source distributions and focus on the surface features of these electricity and magnetism systems are two of the main difficulties associated with these laws. We present this study with a different perspective of observing 322 introductory electricity and magnetism students' understanding of these laws with unconventional shapes for the field sources' enclosing objects. We use a phenomenographic approach to detect difficulties and compare the two contexts. Our main findings suggest that some students make an incomplete or incorrect analysis of the situation when referring to its surface features. The confusions between flux and field in the electric context or between circulation and field in the magnetic context persist. Finally, we present recommendations for teachers to approach these difficulties. 


\section{INTRODUCTION}

A fair amount of research has been conducted regarding conceptual understanding in Electricity and Magnetism (E\&M) [1], focusing on problem-solving ability based on physical principles rather than surface features. In this study, we will be using the surface feature concept to refer to some traits that novice students tend to focus [2]. Research has shown that concepts like Gauss's Law [1, 3-5] and Ampere's Law [6-7] are challenging for undergraduate students, even though instructors prefer to teach them due to their simplicity [8].

These studies have identified possible causes that underlie these difficulties, such as a lack of mathematical tools [6]. Research has shown that, in order to achieve a conceptual understanding of these laws, the students need to develop some abilities, like a) to identify the symmetry between the field and the surface (or trajectory) [4,9-13], b) to move between visual representations $[9,14]$ or $c$ ) to recognize and solve inverted problems [9-10], among others. This study will focus on the difficulty of identification and use of symmetry in Gauss's and Ampere's laws.

Previous studies identified several problems and confusions students have for using symmetry in Gauss's and Ampere's laws. For instance, [4, 10-12] have pointed out that some students try to analyze the symmetry of a Gaussian surface, confusing it with the symmetry of the charge distribution. Studies report that students usually disregard the symmetry and properties of the magnetic field for Ampere's law [13, 15], functionally reducing Ampere's law to a formula (as in, using the equation as a 'magic box' where they 'input' values and get a result as an 'output,' without analyzing its validity). This observation is consistent with studies about other E\&M concepts that arrive at the same conclusion: the systems' surface features often guide students' answers [16-17]. Some studies

\section{(a) Electricity}

The figure shows a point charge $+q$. The figure also shows an imaginary cubic surface enclosing the point charge $+q$. In the figure, the square represents the cubic surface. Using this surface, is it possible to use Gauss's Law to calculate the electric field in any position of the surface? Explain your reasoning.

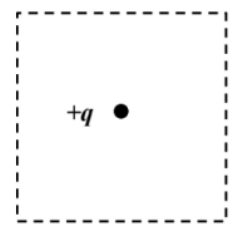

Cubic surface administered open-ended questionnaires, like [12-13, 18], while others used interviews for data collection [7, $10,15]$. It is important to clarify that the most common surface features on which the students of our sample focused are: the form of the enclosing objects; the fact that the surrounding surface or trajectory is closed; the location of the field source; and the form and the symmetry of the shape enclosing the field source.

This study analyzes students' understanding of Gauss's and Ampere's laws by presenting them with problems with similar surface features of representation. We used a set of two problems that involve square-like geometric symmetries and pointlike field sources (a point charge in a cubic surface for Gauss's law and a long, straight wire carrying a conventional current in a square trajectory for Ampere's law). This pair of scenarios provided us relevant insight into students' thoughts when facing unconventional questions (compared to the usual class problems) and difficulties related to these two laws.

In this article, we contribute a detailed comparative analysis of students' understanding about Gauss's and Ampere's laws through problems with parallel surface features. This parallel comparison could point out some ideas for teaching mechanisms that could be applied in both the electricity and magnetism context.

\section{METHODOLOGY}

Our sample has 322 students, all of them enrolled in a calculus-based introductory E\&M course for engineering majors in a large private university in Mexico. We randomly administered each student one of two versions of an open-ended question with similar surface features: one about the identification of symmetry for Gauss's Law (electricity version, Fig. 1(a), 160 students) and the other one about the identification of symmetry for Ampere's Law (magnetism version, Fig. 1(b), 162 students).

\section{(b) Magnetism}

The figure shows a long, straight wire that carries a current $+I_{0}$ with direction out of the page $(\odot)$. The figure also shows an imaginary square trajectory enclosing the current $+I_{0}$. Using this trajectory, is it possible to use Ampere's Law to calculate the magnetic field in any position of the trajectory? Explain your reasoning.

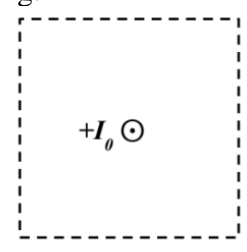

Square Trajectory

FIG 1. (a) Electricity version of the question, with a cubic surface around a point charge, administered to 160 students. (b) Magnetism version of the question, with a square trajectory around a long, straight wire that carries a conventional current, administered to 162 students. 
To answer each question, students need first to analyze that the field is radial in the electricity case and circular in the magnetism case. Also, the students should identify that the imaginary enclosing object has a square-like symmetry (a cube in the electricity version and a square in the magnetism question). The students should acknowledge that the field is not the same magnitude in all positions of each of the enclosing imaginary objects and or that the angle between the field and the surface vector is not constant. Finally, students must conclude that it is impossible to use the given surface or trajectory to calculate the field. We conducted the study in Spanish.
We present a translation of the instrument and students' answers.

We use a phenomenographic approach to analyze the results. The procedure was to create categories from a 20-students random sample, reaching a consensus between experts; then, the two authors analyzed the rest of the answers and classified them into those categories. If more categories emerged, we included them in the analysis in an iterative process. We finally used Cohen's kappa for analysis reliability, with a 0.96 value for the electricity version and a 0.95 value for the magnetism counterpart, above the acceptable value according to the literature [19].

TABLE I. We present the categories and frequency in each context. Below the category's description, we provide an example of an answer per each version of the problem.

\begin{tabular}{|c|c|c|c|}
\hline Category & Reasoning description & Electricity & Magnetism \\
\hline $\begin{array}{l}\text { Evaluates field } \\
\text { and imaginary } \\
\text { enclosing object }\end{array}$ & $\begin{array}{l}\text { The student evaluates if the presented scenario meets the required } \\
\text { conditions for using the laws by focusing on the symmetry of the } \\
\text { field and the imaginary enclosing object. } \\
\text { "No. The field is not perpendicular to the surface." } \\
\text { "Not possible. The magnetic field is not the same at all points of } \\
\text { the trajectory." }\end{array}$ & $11 \%$ & $11 \%$ \\
\hline $\begin{array}{l}\text { Evaluates only on } \\
\text { imaginary } \\
\text { enclosing object }\end{array}$ & $\begin{array}{l}\text { The student evaluates only the surface geometrical features of the } \\
\text { imaginary enclosing object. } \\
\text { "It is not possible because the surface is not a sphere." } \\
\text { "It is not possible because the current is not in a circular } \\
\text { trajectory." }\end{array}$ & $19 \%$ & $18 \%$ \\
\hline $\begin{array}{l}\text { Identifies only an } \\
\text { enclosed field } \\
\text { source }\end{array}$ & $\begin{array}{l}\text { The student focuses only on the fact that the field source is } \\
\text { enclosed in an imaginary object, disregarding the need for a } \\
\text { specific configuration. } \\
\text { "Yes, because it is a charge inside a gaussian surface." } \\
\text { "Yes, because it is a current in the center of a closed trajectory." }\end{array}$ & $22 \%$ & $19 \%$ \\
\hline $\begin{array}{l}\text { Turns to an } \\
\text { authority fallacy }\end{array}$ & $\begin{array}{l}\text { The student states that Gauss's or Ampere's laws could be applied } \\
\text { because of an authority fallacy. } \\
\text { "Yes, because that is what Gauss's law is for." } \\
\text { "Yes, because Ampere's law is used for these cases." }\end{array}$ & $19 \%$ & $16 \%$ \\
\hline $\begin{array}{l}\text { Focuses only on } \\
\text { geometric } \\
\text { symmetry }\end{array}$ & $\begin{array}{l}\text { The student focuses on the geometry of the imaginary enclosing } \\
\text { object. Since the square or cube is symmetrical, the student } \\
\text { claims that the laws serve to calculate the field. } \\
\text { "Yes, because there is symmetry in the surrounding surface." } \\
\text { "Yes, because the trajectory is symmetrical." }\end{array}$ & $6 \%$ & $6 \%$ \\
\hline $\begin{array}{l}\text { Indicates it is } \\
\text { very complicated }\end{array}$ & $\begin{array}{l}\text { The student claims that they could use the law to calculate the } \\
\text { electric field using a more complex mathematical treatment. } \\
\text { "Yes, but it would be complicated to compute." } \\
\text { "Yes, but the calculations would be very complicated." }\end{array}$ & $5 \%$ & $6 \%$ \\
\hline Other & Unclassifiable answers & $14 \%$ & $20 \%$ \\
\hline Unanswered & Students who left the question totally blank. & $4 \%$ & $4 \%$ \\
\hline Total & & $100 \%$ & $100 \%$ \\
\hline
\end{tabular}




\section{RESULTS AND DISCUSSION}

The same six main categories emerged from the two questions, with very similar results on both versions. Table I presents the categories along with a brief description. We want to point out that all categories appeared for the two contexts symmetrically, implying remarkable parallelism between the two laws regarding students' understanding. The results are very similar in frequency, with differences of around 3\% or less for all six main categories between electricity and magnetism results. Moreover, the "Evaluates field and imaginary enclosing object" category (which we consider the complete answer) grouped the same percentage of students in both contexts.

The category "Evaluates only on imaginary enclosing object" shows that some students may provide correct answers without referring to physical principles. For instance, it is not wrong to express that Gauss's law cannot be used because of the cubic surface, but $19 \%$ of the students did not express why the surface's shape matters, thus providing no evidence of complete understanding of Gauss's law. The same goes for the $18 \%$ of students who answer Ampere's law scenario similarly. More likely, this may suggest that students are focusing on surface features instead of the properties of the fields, as reported by other studies [7, 15-17]. We consider the shape of the imaginary enclosing object or the mere fact that the scenario presents a centered, enclosed field source inside such imaginary enclosing object as surface features. Also, we would like to point out that this might as well be memorization of the "adequate" imaginary enclosing object, in which case the student would still be focusing on a surface feature. We consider this category partially correct because students conclude that they cannot use the marked surface or trajectory to calculate the field using Gauss's or Ampere's law. However, it is relevant to acknowledge that the students in this category refer to surface features of the problem instead of the underlying principles. We do not have enough evidence to confirm that their understanding of Gauss's or Ampere's laws is complete -opposite of what happens in the following two categories.

The category "Identifies only an enclosed field source" grouped students stating that it is possible to calculate the field with the shown surface or trajectory, basing their answers on surface features. Most of the answers focused only on the enclosing object being closed, while others added that the field source is at the center. This difficulty was most frequent in both versions of the question, with $22 \%$ for electricity and $19 \%$ for magnetism.
The category "Focuses only on geometrical symmetry" was consistent in both contexts, with $6 \%$ of the students in each version of the problem. This category provides evidence that these students may confuse the symmetry of the field and its enclosing object (which would then allow simple calculations through either of the laws) and the geometric meaning of symmetry, which is visualized in the imaginary enclosing object. Other studies have also reported a similar confusion $[4-5,10]$, and it could imply that some students know that some symmetry is necessary to use Gauss's or Ampere's laws correctly. However, the lack of understanding of the meaning of symmetry in the context of E\&M makes them turn to the more familiar geometric term, which is also a characteristic that they can see, reinforcing the idea.

Finally, the category "Other" groups unclassifiable answers. Some of them were initially grouped into categories of their own. However, if their frequency was below 5\%, we considered them not representative and classified them into the "Other" category.

The three categories above have the common denominator of surface features. We observe that less than $20 \%$ of students may use surface features to reach a correct conclusion without analyzing if the required physical conditions are met in the category "Evaluates only on imaginary enclosing object" in both contexts. However, the other two categories related to the problem's surface features add up to over $25 \%$ of students who conclude that using the shown surface or trajectory to calculate the field with Gauss's or Ampere's law is possible.

In the category "Turns to an authority fallacy," we observe that students evoked an authority fallacy: because Gauss's and Ampere's laws are physical laws, they are always valid. Therefore, it should be possible to calculate the field using them in any situation. To make this statement evident, we even encountered answers such as "yes, because Gauss's law is almighty." Studies like [11] and [13] point out that some students functionally reduce these laws to formulas applied to every scenario. We think this idea could be the basis of the categories "Identifies only an enclosed field source" and "Turns to an authority fallacy." In other words, as one student stated, "that is what Gauss's [or Ampere's] law is for."

The last category was "Indicates it is very complicated," with 5\% of the student sample following this reasoning in the electricity problem and $6 \%$ doing so in the magnetism problem. The students in this category turn to the idea of a very complex mathematical process that could be beyond the tools they understand (just as [6] mentioned). Similar results have been reported previously, for example, in [10], where some students described the needed mathematical process as messy but not impossible. 
The two categories above, "Turns to an authority fallacy" and "Indicates it is very complicated," may imply a naïve interpretation of electric flux or magnetic circulation, leading to confusion between electric flux and electric field, or magnetic circulation and magnetic field. For instance, Gauss's law describes the electric flux on any Gaussian surface. Since some students cannot tell the difference between electric flux and electric field, they assume Gauss's law works in every case to calculate an electric field. The same inference could be made with Ampere's law and the magnetic circulation. In the category "Indicates it is very complicated," we think that students focus on the left side of Gauss's and Ampere's law, which is the definition of flux and circulation, respectively, implying a slightly distinct confusion between electric flux and field, or magnetic circulation and field.

Overall, the results show that there are persistent difficulties in using Gauss's and Ampere's laws regardless of the contexts. The use of surface features in these problems provided information on how many students build their answers. As Table I shows, a fraction of the students concurred in a partially correct answer (in the category "Evaluates only on imaginary enclosing object," with $19 \%$ of the students for electricity and $18 \%$ for magnetism). However, a more prominent segment of the sample produced an incorrect conclusion (in the categories "Identifies only an enclosed field source" and "Focuses only on geometrical symmetry," with a combined $28 \%$ in electricity and $25 \%$ in magnetism).

This finding points out the need to implement different scenarios where students must identify physical symmetry and analyze the necessary conditions to compute the electric or magnetic field using Gauss's and Ampere's laws. The different scenarios need to include inverted problems, presenting cases in which calculating the field is impossible, such as the one shown in this contribution.

\section{CONCLUSIONS AND TEACHING SUGGESTIONS}

Our data suggest Gauss's and Ampere's laws are challenging concepts for E\&M undergraduate students, with just above 1 out of 10 students demonstrating a complete conceptual understanding. Most of the students seem to reduce these laws to formulas, disregarding any characteristic of the field or their source's distribution. The surface features seem to impact students' answers and reasoning as well.

The parallel comparison allowed the identification of difficulties that emerge similarly from both E\&M concepts. The use of similar surface features to represent problems for symmetry analysis and the phenomenographic technique used in this study provided a glimpse of the need for a unified approach on student difficulties in the conceptual understanding of Gauss's and Ampere's laws. This information could be taken as an advantage since the difficulties found are the same for both contexts, allowing similar approaches during instruction.

We could express three teaching suggestions that emerge from these findings. We encourage instructors to present students with some conceptual problems where they are required to analyze the symmetry of field source distributions and the election of the proper enclosing object. Subsequently, the calculation of the field could be computed. Moreover, we suggest instructors explicitly include cases where Gauss's and Ampere's laws do not help compute the field, in addition to the traditional problems where they do. The teaching practice must allow the students to understand that Gauss's and Ampere's laws are, evidently, laws and apply to all the physical situations. However, they are not always helpful in calculating the electric or magnetic field. There is a need for more research that specifically addresses the impact of surface features in the conceptual understanding and use of Gauss's and Ampere's laws.

As a second suggestion, we encourage teachers to be explicit on the scope of Gauss's and Ampere's laws since this might raise awareness in the students when facing such problems.

Finally, we recommend teachers to emphasize the mathematical simplicity that comes from the correct symmetry analysis. Gauss's and Ampere's laws can be used to simplify problems in E\&M. Instructors may develop similar parallel activities to address the difficulties for understanding these laws.

As suggested in this study, it is also necessary to research students' confusion between electric flux and electric field and the confusion between magnetic circulation and magnetic field. Our data provide evidence that this confusion may exist, but this analysis is beyond the scope of this article. Future studies must address this explicitly.

The type of analysis that we present in this study, where we can compare students' understanding of the symmetry conditions to use Gauss's and Ampere's laws to calculate the field, contributes to detecting the sources of difficulty that persist in two parallel topics in electricity and magnetism contexts. In this case, we found through the emergent categories that surface features play an essential role. The confusion between electric flux and electric field and magnetic circulation and magnetic field persists. 
[1] D. P. Maloney, T. L. O'Kuma, C. J. Hieggelke, and A. Van Heuvelen, Surveying students' conceptual knowledge of electricity and magnetism, Am. J. Phys. 69, S12 (2001).

[2] Chi, P. Feltovich, and R. Glaser, Categorization and representation of physics problems by experts and novices, Cogn. Sci. 5 (2), 121(1981).

[3] S. Rainson, G. Tranströmer, and L. Viennot, Students' understanding of superposition of electric fields, Am. J. Phys. 69, 11(1994).

[4] C. Singh, Student understanding of symmetry and Gauss's law of electricity. Am. J. Phys. 74, 10(2006).

[5] J. Li and C. Singh, Investigating and improving introductory physics students' understanding of the electric field and superposition principle, Eur. J. Phys. $38,5(2017)$.

[6] J. Guisasola, J. M. Almudí and K. Zuza, The design and evaluation of an instructional sequence on Ampere's law, Am. J. Phys. 78, 11(2010)

[7] C. S. Wallace and S. V. Chasteen, Upper-division students' difficulties with Ampere's law. Phys. Rev. Spec. Top. Phys. Ed. Res. 6, 2(2010).

[8] M. B. Schneider. Discovery-based Gauss's law, Am. J. Phys, 72, 10(2004).

[9] C. A. Manogue, K. Browne, T. Dray, and B. Edwards, Why is Ampère's law so hard? A look at middledivision physics, Am. J. Phys. 74 4(2006)

[10] R. E. Pepper, S. V. Chasteen, S. J. Pollock, and K. K. Perkins. Our best juniors still struggle with Gauss's Law: Characterizing their difficulties. Am. Ins. Phys. $1289,1(2010)$

[11] J. A. Byford, and P. Chahal, Common misunderstandings and challenges in learning Gauss's Law in a junior level electromagnetic engineering course, International Conference on Interactive Collaborative Learning (2017).

[12] J. Li, and C. Singh. Investigating and improving introductory physics students' understanding of symmetry and Gauss's law, Eur. J. Phys. 39, 1(2018).

[13] J. Guisasola, J. M. Almudi, and J. L. Zubimendi, Difficulties in learning the introductory magnetic field theory in the first years of university, Sci. Educ. 88, 443 (2004).

[14] E. Campos, G. Zavala, K. Zuza, and J. Guisasola, Students' understanding of the concept of the electric field through conversions of multiple representations. Phys. Rev. Phys. Ed. Res. 16, 1(2020).

[15] J. Guisasola, J. M. Almudí, J. Salinas, K. Zuza, and M. Ceberio, The Gauss and Ampere laws: different laws but similar difficulties for student learning. Eur. J. Phys. 29 5(2008)

[16] T. M. Scaife and A. F. Heckler, Interference between electric and magnetic concepts in introductory physics, Phys. Rev. ST Phys. Educ. Res. 7, 010104 (2011).

[17] E. Hernandez, E. Campos, P. Barniol, and G. Zavala,. The effect of similar surface features on students' understanding of the interaction of charges with electric and magnetic fields. PERC Proc. 2019, (2020)

[18] J. Guisasola, J. Salinas, J. M. Almudí, and S. Velazco, Analysis of the processes of application of Gauss's and Ampere's Laws by university students in Spain and Argentina. Rev. Bra. Ens. Fis. 25, 2(2003).

[19] K. Zuza, P. van Kampen, M. De Cock, T. Kelly, and J. Guisasola, Introductory university physics students' understanding of some key characteristics of classical theory of the electromagnetic field, Phys. Rev. Phys. Educ. Res. 14, 020117 (2018). 